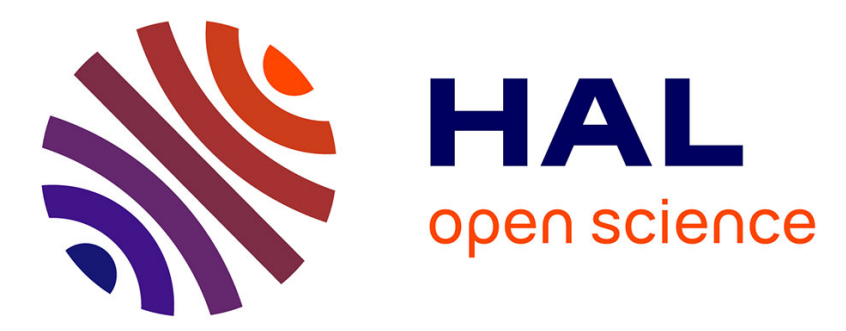

\title{
Controllable quantities for bilinear quantum systems
}

Gabriel Turinici

\section{To cite this version:}

Gabriel Turinici. Controllable quantities for bilinear quantum systems. Control and Decision Conference, 2000, Sydney, Australia. pp.1364-1369. hal-00723667

\section{HAL Id: hal-00723667 \\ https://hal.science/hal-00723667}

Submitted on 8 Mar 2013

HAL is a multi-disciplinary open access archive for the deposit and dissemination of scientific research documents, whether they are published or not. The documents may come from teaching and research institutions in France or abroad, or from public or private research centers.
L'archive ouverte pluridisciplinaire HAL, est destinée au dépôt et à la diffusion de documents scientifiques de niveau recherche, publiés ou non, émanant des établissements d'enseignement et de recherche français ou étrangers, des laboratoires publics ou privés. 


\title{
Controllable quantities for bilinear quantum systems
}

\author{
Gabriel TURINICI \\ ASCI-CNRS Laboratory, Bat. 506, Université Paris Sud, 91405 Orsay Cedex \\ turinici@asci.fr
}

\begin{abstract}
This paper is dedicated to the search of tailored controllability concepts for quantum systems interacting with lasers. A negative result for infinite dimensional spaces serves as motivation for a finite dimensional analysis. We show that under physically reasonable hypothesis we can locally control sets of observables. As a remarkable particular case global exact controllability is proven for the population of the eigenstates.
\end{abstract}

\section{Introduction}

Controlling chemical reactions at the quantum level was a long-lasting goal $[1,3,4,5,9,11,13,14,16,17]$ from the very beginning of the laser technology. Indeed, due to the subtle nature of the interactions involved, this kind of manipulation is expected to allow on the one hand for much efficient and finer control than classical tools and on the other hand for new phenomena to be revealed.

The first experiments have shown that designing the laser pulse able to ensure the desired properties of the system is a non-trivial task that physical intuition alone cannot accomplish. It is only recently that tools coming from the control theory began to give satisfactory results in some particular cases.

A legitimate question arises in this context: what are the new controllability concepts that best fit this framework and which are the quantum quantities that can be exactly controlled using such an external field ? Some answers are given below.

\section{Infinite dimensional controllability}

The problem under study is controlling the time evolution of quantum systems. Let us consider such a independent system with internal Hamiltonian $H_{0}$ and let $\Psi_{0}(x)$ be its initial state. Denoting by $\Psi(x, t)$ the state at the time $t$ the evolution equations (Time Dependent
Schrödinger Equations) for the free system read:

$$
\left\{\begin{array}{l}
i \hbar \frac{\partial}{\partial t} \Psi(x, t)=H_{0} \Psi(x, t) \\
\Psi(x, t=0)=\Psi_{0}(x),\left\|\Psi_{0}\right\|_{L^{2}\left(\mathbf{R}^{\gamma}\right)}=1
\end{array}\right.
$$

The external action expected to allow for control is a laser field modeled by a laser intensity $\epsilon(t) \in \mathbf{R}$ and by a certain time independent dipole moment operator $\mathcal{B}$ (see also [18]). The new Hamiltonian is $H=H_{0}-\epsilon(t) \mathcal{B}$ and the dynamical equations read:

$$
\left\{\begin{array}{l}
i \hbar \frac{\partial}{\partial t} \Psi_{\epsilon}(x, t)=H \Psi_{\epsilon}(x, t) \\
\Psi_{\epsilon}(x, t=0)=\Psi_{0}(x)
\end{array}\right.
$$

In a first approximation the goal may be formalized as to find (if any) a final time $T$ and a finite energy laser pulse $\epsilon(t), \epsilon(t) \in L^{2}([0, T])$ able to steer the system from $\Psi_{0}(x)$ to some predefined target $\Psi_{\epsilon}(x, T)=\Psi_{\text {target }}(x)$.

Note that the $L^{2}$ norm of $\Psi_{\epsilon}$ is conserved throughout the evolution:

$$
\left\|\Psi_{\epsilon}(x, t)\right\|_{L_{x}^{2}\left(\mathbf{R}^{\gamma}\right)}=\left\|\Psi_{0}\right\|_{L^{2}\left(\mathbf{R}^{\gamma}\right)}, \forall t>0 .
$$

In general, for any autoadjoint operator $O$ such that $\left[H_{0}, O\right]$ and $[\mathcal{B}, O]$ are both zero [19] one obtains

$$
<\Psi_{\epsilon}(x, t)|O| \Psi_{\epsilon}(x, t)>=<\Psi_{0}|O| \Psi_{0}>, \forall t>0,
$$

with the usual notation $\langle\Psi|O| \Psi\rangle=\langle\Psi, O \Psi\rangle_{L^{2}}=<$ $O \Psi, \Psi>{ }_{L^{2}}$. One remarkable class of operators are $L^{2}-$ projections to closed subspaces. Let $P$ be a projection to a closed subspace $X$ of $L^{2}\left(\mathbf{R}^{\gamma}\right)$. Then $\left[H_{0}, P\right]=$ $[\mathcal{B}, P]=0$ mean in particular that $X$ and its orthogonal complement $X^{\perp}$ are involutive for $H_{0}$ and $\mathcal{B}$, i.e.

$$
\left\{\begin{array}{l}
\forall \Psi \in X: H_{0} \Psi \in X, \mathcal{B} \Psi \in X \\
\forall \Psi \in X^{\perp}: H_{0} \Psi \in X^{\perp}, \mathcal{B} \Psi \in X^{\perp}
\end{array}\right.
$$

The system can then be viewed as decomposed into two independent subsystems with wavefunctions the projections of the total wavefunction to $X$ and $X^{\perp}$. Of course this decomposition can be further refined for any additional projection operator that commutes with $H_{0}$ and $\mathcal{B}$. In order not to introduce unnecessary complications, we will suppose in all that follows that the system has only a finite number of independent subsystems (although the theory can be accommodated to fit a countable number of subsystems which can be proved to be 
the general case), each being associated its $L^{2}$-projector $P_{1}, \ldots, P_{K}$ such that:

$$
\left[H_{0}, P_{i}\right]=\left[\mathcal{B}, P_{i}\right]=0, \forall i=1, \ldots, K
$$

Moreover one can prove that the projectors can be chosen to fulfill the following conditions:

$$
\sum_{i=1}^{K} P_{i}=I, P_{i} P_{j}=0, \forall i \neq j, i, j=1, \ldots K
$$

Denote by $S_{\Psi_{0}}$ the product of hyper-spheres: $S_{\Psi_{0}}=$ $\left\{f \in L^{2}\left(\mathbf{R}^{\gamma}\right) ;\left\|P_{i} f\right\|_{L^{2}\left(\mathbf{R}^{\gamma}\right)}=\left\|P_{i} \Psi_{0}\right\|_{L^{2}\left(\mathbf{R}^{\gamma}\right)}, i=\right.$ $1, \ldots, K\}$ By using 2.4 for the projectors $P_{1}, \ldots, P_{k}$ one can prove that the system evolves on $S_{\Psi_{0}}$.

Let us point out that due to the quantum nature of the system it follows by the uncertainty principle that one will never be able to experimentally verify, neither fully exploit, the exact controllability. In fact even if one obtains exactly the desired target state $\Psi_{\text {target }}$ the free evolution (i.e. when laser is switched off $\epsilon(t)=0, t \geq T$ ) of the quantum system instantaneously modifies this state (by a time dependent phase shift if $\Psi_{\text {target }}$ is an eigenfunction of $H_{0}$ and by the (2.1) formula in general). In this context a negative controllability result is therefore not really restrictive. In fact using arguments as in [2] we may prove (see also [20]) :

Theorem 2.1 Let $\mathcal{B}$ be a bounded operator from $H_{x}^{2}\left(\mathbf{R}^{\gamma}\right)$ to itself and let $H_{0}$ generate a $C^{0}$ semigroup of bounded linear operators on $H_{x}^{2}\left(\mathbf{R}^{\gamma}\right)$. Denote by $\Psi_{\epsilon}(x, t)$ the solution of (2.2). Then the set of attainable states from $\Psi_{0}$ defined by

$$
\mathcal{A S}=\cup_{T>0}\left\{\Psi_{\epsilon}(x, T) ; \epsilon(t) \in L^{2}([0, T])\right\}
$$

is contained in a countable union of compact subsets of $H_{x}^{2}\left(\mathbf{R}^{\gamma}\right) \cap S_{\Psi_{0}}$. In particular its complement with respect to $S_{\Psi_{0}}: \mathcal{N}=S_{\Psi_{0}} \backslash \mathcal{A S}$ is everywhere dense on $S_{\Psi_{0}}$. The same holds true for the complement with respect to $S_{\Psi_{0}} \cap H_{x}^{2}\left(\mathbf{R}^{\gamma}\right)$.

Proof: To prove the first part of the theorem one applies Thm. 3.6 from [2] on the space $H_{x}^{2}\left(\mathbf{R}^{\gamma}\right)$ for the operators $-i H_{0}$ and $-i \mathcal{B}$ (and restricts $\epsilon(t)$ to $L^{2}$ functions). Denote for any set $A$ :

$$
A_{r_{1}, \ldots, r_{K}}=\left\{\sum_{i=1}^{K} s_{i} P_{i} f ; 0 \leq s_{i} \leq r_{i}, f \in A\right\}
$$

Then for any compact subset $C$ of $X C_{r_{1}, \ldots, r_{K}}$ is also compact. Applying this to the compact components $C$ of $\mathcal{A S}$ one notes that

$$
\cup_{r_{1} \geq 0, \ldots, r_{K} \geq 0} \mathcal{A} \mathcal{S}_{r_{1}, \ldots, r_{K}}=\cup_{n \in \mathbf{N}^{*}} \mathcal{A} \mathcal{S}_{n, \ldots, n}
$$

is also a countable union of compacts subsets of $H_{x}^{2}\left(\mathbf{R}^{\gamma}\right)$. It follows by the Baire category theorem that $\cup_{r_{1} \geq 0, \ldots, r_{K} \geq 0} \mathcal{A S}_{r_{1}, \ldots, r_{K}}$ has dense complement in $H_{x}^{2}\left(\mathbf{R}^{\gamma}\right)$; in particular the complement of $\mathcal{A S}$ with respect to $S_{\Psi_{0}} \cap H_{x}^{2}\left(\mathbf{R}^{\gamma}\right)$ has to be everywhere dense on $S_{\Psi_{0}} \cap H_{x}^{2}\left(\mathbf{R}^{\gamma}\right)$.

Given this result the search for exactly controllable quantities has to be directed to the finite dimensional setting.

\section{Finite dimensional controllability}

Let $D=\left\{\Psi_{i}(x) ; i=1, . ., N\right\}$ be an orthonormal basis for a finite dimensional sub-space $F$ of $L^{2}\left(\mathbf{R}^{\gamma}\right)[21]$ and $A$ and $B$ be the matrices of the operators $H_{0}$ and $\mathcal{B}$ with respect to this base.

Denote $C=\left(c_{i}\right)_{i=1}^{N}$ as the coefficients of $\Psi_{i}(x)$ in the formula of the evolving state $\Psi(x, t)=\sum_{i=1}^{N} c_{i}(t) \Psi_{i}(x)$. From now we will work in atomic units only $(\hbar=1)$; the equations (2.2) read

$$
\begin{array}{r}
i \frac{\partial}{\partial t} C_{\epsilon}=A C_{\epsilon}-\epsilon(t) B C_{\epsilon}, C_{\epsilon}(t=0)=C_{0} \\
C_{0}=\left(c_{0 i}\right)_{i=1}^{N}, c_{0 i}=<\Psi_{0}, \Psi_{i}>
\end{array}
$$

The controllability of (3.1) has been dealt with in the literature (cf. [12]) by deriving results from the controllability of a system posed on the space of the unitary matrices of dimension $N$. This approach has the benefit of granting access to the general tools on the controllability of bilinear systems on Lie groups. However, these results give only sufficient conditions for exact controllability (due to the setting which is more general). Finally there exists a class of simple quantum systems controllable in a sense to be defined further on that do not verify the criteria emerging from the Lie group analysis. We have therefore judged instructive to study this issue in a new framework; we were thus lead into identifying simple necessary and sufficient conditions for the finite dimensional controllability (see also [5] for an introduction to this topic).

In the case of our modeling the $A$ matrix is diagonal and $B$ is symmetrical with null diagonal elements (see [15] for the general case). Let us denote by $\lambda_{i}, i=1, . ., N$ the diagonal elements of $A$ (the energies of the states $\left.\Psi_{i}\right)$. Before presenting the theoretical results we will introduce the controllability concept used.

Let $O_{1}, \ldots, O_{p}$ be positive quantum observables (positive autoadjoint operators). We say that the distribution of observables $\delta=\left(\delta_{i}\right)_{i=1}^{p}, \delta_{i} \geq 0, i=1, \ldots, p$ is reachable from the initial state $C_{0}$ if for any $\eta>0$ there exists a final time $T_{d}>0$ and an electric field $\epsilon(t) \in L^{2}\left(\left[0, T_{d}\right]\right)$ such that the solution of (3.1) satisfies:

$$
\left|<\Psi\left(x, T_{d}\right)\right| O_{i}\left|\Psi\left(x, T_{d}\right)>-\delta_{i}^{2}\right|<\eta, i=1, \ldots, p
$$


If this is also true for $\eta=0$ we say that the distribution of observables $\delta$ can be exactly reached from the initial state $C_{0}$.

A special case of positive observables are the projections on the eigenstates $P_{\Psi_{i}}$ defined by $P_{\Psi_{i}} \Psi=<\Psi, \Psi_{i}>_{L^{2}}$ $\Psi_{i}, i=1, \ldots N$. The observable quantities $\left\langle\Psi\left|P_{\Psi_{i}}\right| \Psi\right\rangle$ corresponding to this operators are called populations of the eigenstates. In our case these are $\left|c_{\epsilon k}\left(T_{d}\right)\right|^{2}$. A remarkable property of these observables is that when the system is evolving freely $((3.1)$ with $\epsilon(t)=0)$ the populations of the eigenstates do not change.

As it was previously seen the system evolves on the unit sphere of $L_{x}^{2}\left(\mathbf{R}^{\gamma}\right)$ which in finite dimensional representation reads $\sum_{i=1}^{N}\left|c_{\epsilon i}(t)\right|^{2}=1, \forall t \geq 0$. We call population distribution for the system (3.1) any $N$-tuple $d \in \mathbf{R}^{N}$ such that

$$
\sum_{i=1}^{N} d_{i}^{2}=1, d_{i} \geq 0, i=1, \ldots, N
$$

A population distribution being a particular case of distribution of observables we extend the reachability concepts defined above to this case also.

\section{Transfer graph and necessary conditions}

We define as in [15] the non-oriented transfer graph of the system $G=(V, E)$ which corresponds to the intuitive image of population flow among different eigenstates of the system. The set $V$ of vertices is the set of eigenstates $\Psi_{i}$ and the set of edges $E$ is the set of all pairs of eigenstates coupled by the matrix $B$ :

$G=(V, E), V=\left\{\Psi_{1}, \ldots, \Psi_{N}\right\} E=\left\{\left(\Psi_{i}, \Psi_{j}\right) ; B_{i j} \neq 0\right\}$

This graph can be decomposed into connected components $G_{\alpha}=\left(V_{\alpha}, E_{\alpha}\right), a=1, . ., K$ that correspond to a bloc-diagonal structure of the matrix B (modulo permutations on the indices). It is worthwhile mentioning that this operation is the discrete version of the decomposition using projection operators that was undertaken for the infinite dimensional case; indeed, for each connected component $G_{\alpha}, \alpha=1, \ldots, K$, one can associate the linear space spanned by the eigenfunctions in $V_{\alpha}$ and prove that the (discrete) projection operator on this space $P_{\alpha}$ commutes with $A$ and $B$.

Let $\tilde{D}=\left\{\tilde{\Psi}_{1}, \ldots, \tilde{\Psi_{N}}\right\}$ be an orthonormal basis for the finite dimensional space $F$ and $\tilde{P}_{1}, \ldots, \tilde{P}_{N}$ projections operators on $\tilde{\Psi}_{1}, \ldots, \tilde{\Psi}_{N}$ respectively. Suppose moreover that these observables are commuting with $P_{1}, \ldots, P_{K}$, which is equivalent to the fact that $\tilde{D}$ is the union of orthonormal basis for each subsystem. Denote by $U$ the unitary matrix that allow to change between the orthonormal basis $D$ and $\tilde{D}: \tilde{\Psi}_{i}=\sum_{j} U_{i j} \Psi_{j}$. We will suppose in all that follows that all entries in $U$ are real.

One can check by the definition of $G$ and using equations (3.1) that for all $\alpha=1, . ., K: i \frac{d}{d t}\left\|P_{\alpha} \Psi(x, t)\right\|_{L^{2}}^{2}=$ 0 ; each subsystem (connected component) comply therefore with the conservation laws

$$
\begin{aligned}
\sum_{\left\{i ; \Psi_{i} \in V_{\alpha}\right\}}<\Psi(x, t)\left|\tilde{P}_{i}\right| \Psi(x, t) & >=\text { constant } \\
t & >0, \alpha=1, . ., K
\end{aligned}
$$

This allows us to give necessary conditions for controllability

Lemma 4.1 If the distribution of observables $\delta$ is reachable from the initial configuration $C_{0}$ then

$$
\sum_{\left\{i ; \Psi_{i} \in V_{\alpha}\right\}}<\Psi_{0}\left|\tilde{P}_{i}\right| \Psi_{0}>=\sum_{\left\{i ; \Psi_{i} \in V_{\alpha}\right\}} \delta_{i}^{2}, \alpha=1, . ., K .
$$

As a particular case one obtains the following

Corollary 4.1 If the population distribution $d$ is reachable from the initial configuration $C_{0}$ then

$$
\sum_{\left\{i ; \Psi_{i} \in V_{\alpha}\right\}}\left|c_{0 i}\right|^{2}=\sum_{\left\{i ; \Psi_{i} \in V_{\alpha}\right\}} d_{i}^{2}, \quad \alpha=1, . ., K .
$$

\section{Controllability results}

Denote $\omega_{k l}=\lambda_{k}-\lambda_{l}, k, l=1, \ldots, N$. Let us introduce the following hypothesis:

$\mathbb{H} \mathbb{A}$ The components $G_{\alpha}, \alpha=1, . ., K$ of $G$ remain connected after elimination of all edge pairs $\left(\Psi_{i}, \Psi_{j}\right),\left(\Psi_{a}, \Psi_{b}\right)$ such that $\omega_{i j}=\omega_{a b}$ (degenerate transitions).

Theorem 5.1 (Local exact controllability) Let $T>0$ be a given final time, $\epsilon_{0}(t) \in L^{2}([0, T])$ a given laser field such that:

$\mathbb{H} \mathbb{B} \lim _{t \rightarrow T} \epsilon_{0}(t)=0$

so in particular the limit $\lim _{t \rightarrow T} \epsilon_{0}(t)$ is supposed to exist (see also Remark 5.1); let $\Psi_{T}$ be the state at time $T$ of the system propagated with the laser field $\epsilon_{0}$ and $\delta_{T}\left(d_{T}\right)$ the distribution of observables (populations) associated to the state $\Psi_{T}$ :

$$
\begin{gathered}
\delta_{T}=\left(\sqrt{<\Psi_{T}\left|\tilde{P}_{i}\right| \Psi_{T}>}\right)_{i=1}^{N}, \\
d_{T}=\left(\left|<\Psi_{T}, \Psi_{i}>_{L^{2}}\right|\right)_{i=1}^{N}=\left(\left|c_{0 i}\right|\right)_{i=1}^{N} .
\end{gathered}
$$


Suppose $\left(d_{T}\right)_{i} \neq 0,\left(\delta_{T}\right)_{i} \neq 0, i=1, \ldots, N$ and that the hypothesis $\mathbb{H} \mathbb{A}$ is verified. Suppose also that:

$\mathbb{H} \mathbb{C}$ For each connected component $G_{\alpha}, \alpha=1, \ldots, K$ of $G$ it does not exists a partition $V_{\alpha}=V_{\alpha}^{1} \cup V_{\alpha}^{2}, V_{\alpha}^{1} \cap$ $V_{\alpha}^{2}=\emptyset$ such that

$\left|\sum_{a \in V_{\alpha}^{1}} U_{j q}<\Psi_{T}, \Psi_{a}>\right|=\left|\sum_{b \in V_{\alpha}^{2}} U_{j b}<\Psi_{T}, \Psi_{b}>\right|, \forall j \in V_{\alpha}$

or if such a partition exists then

$$
\frac{\sum_{a \in V_{\alpha}^{1}} U_{j a}<\Psi_{T}, \Psi_{a}>}{\sum_{b \in V_{\alpha}^{2}} U_{j b}<\Psi_{T}, \Psi_{b}>}=\text { constant }, \forall j \in V_{\alpha} .
$$

Then there exists an open neighborhood $D$ of $\delta_{T}$ on the surface of $\mathbf{R}^{N}$ given by the necessary conditions (4.3) endowed with the canonical topology such that one can exactly reach any distribution of observables $\delta$ in $D$ from $C_{0}$.

Remark 5.1 The hypothesis $\mathbb{H} \mathbb{B}$ is not really restrictive. In all practical cases $\epsilon_{0}(t)$ is continuous (at least at final/initial time) which assures the existence of the limit. The requirement that the limit of $\epsilon_{0}(t)$ in $T$ be exactly 0 can be readily satisfied by replacing the triplet $\left(\epsilon_{0}, A, B\right)$ by $\left(\epsilon-\epsilon_{0}(T), A+\epsilon_{0}(T) B, B\right)$, where $\epsilon_{0}(T)=\lim _{t \rightarrow T} \epsilon_{0}(t)$. Note that in this situation the hypothesis $\mathbb{H} \mathbb{A}$ has to be verified for the eigenvalues of $A+\epsilon_{0}(T) B$ which are in general different from those of A. Finally, note that the set of final states $\Psi_{T}$ that do not comply with the hypothesis $\mathbb{H} \mathbb{C}$ is of null canonical measure for any (real) unitary matrix $U$.

Remark 5.2 The result above may be somehow surprising due to the specific concept of locality used. In fact, suppose that the evolution of the system has ended in some final state $p_{T}$ with the corresponding distribution of observables $\delta_{T}$. Then, in order to obtain some other admissible distribution $\delta_{c}$ close to $\delta_{T}$ one has to go back in time and modify the electric field rather than to start from $p_{T}$ and go for $\delta_{c}$ ! To understand this one has to remember that the observables do not necessarily commute with the hamiltonian so the free evolution (from $p_{T}$ ) drags the distribution of observables towards the direction given by the evolution equations 2.2; there is therefore no reason to hope that small perturbations (after the time $T$ ) can always counter-balance this bias and at the same time fill out a neighborhood of $\delta_{T}$.

Remark 5.3 The technical conditions $\left(\delta_{T}\right)_{i} \neq 0, i=$ $1, \ldots, N$ can also be intuitively justified. Indeed if some $\left(\delta_{T}\right)_{i}=0$ one have to take care when choosing the good target set to expect exact controllability into, since there is no reason to hope in (exactly) reaching "distributions" having some strictly negative observables, as any projection-like observable is a positive operator.

Proof: For the sake of simplicity we treat only the case $\omega_{i j} \neq \omega_{a b}, \forall(i, j) \neq(a, b)$, the general case bearing no new concepts. Let us denote $\bar{A}=-i A$ and $\bar{B}=$ $-i B$. Then (3.1) become:

$$
\frac{\partial}{\partial t} C_{\epsilon}=(\bar{A}+\epsilon(t) \bar{B}) C_{\epsilon}, \quad C_{\epsilon}(t=0)=C_{0}
$$

Denote by $c\left(\epsilon, C_{0}, t\right)=\left(c_{a}\left(\epsilon, C_{0}, t\right)\right)_{a=1}^{N}$ the solution at the time $t$ of (3.1) for the initial $(t=0)$ data $C_{0}$ and electric field $\epsilon(t)$. Denote also $w(t)=c\left(\epsilon_{0}, C_{0}, t\right)$ and consider the canonical base $\left\{e_{1}, \ldots, e_{N}\right\}$ of $\mathbf{R}^{N}$.

We define the application $M: L^{2}(\mathbf{R}) \rightarrow \mathbf{R}^{N}$ given by

$$
M(\epsilon)=\left(<c\left(\epsilon, C_{0}, T\right)\left|\tilde{P}_{a}\right| c\left(\epsilon, C_{0}, T\right)>\right)_{a=1}^{N}
$$

Note that by the necessary conditions (4.3) the range of $M$ is a subset of

$$
\begin{array}{r}
\left\{\left(x_{i}\right)_{i=1}^{N} \in \mathbf{R}^{N} ; \sum_{\left\{i ; \Psi_{i} \in V_{\alpha}\right\}} x_{i}=\sum_{\left\{i ; \Psi_{i} \in V_{\alpha}\right\}}<\Psi_{0}\left|\tilde{P}_{i}\right| \Psi_{0}>\right. \\
\alpha=1, . ., K\}
\end{array}
$$

The local controllability is in fact a particular surjectivity property of $M$. We will prove that the differential $D M$ of $M$ has the surjectivity property we desire and by the implicit function theorem the conclusion will follow then for $M$ itself. More precisely we prove that $D M$ is onto the linear manifold $(\mathrm{P})$ (product of hyper-planes of $\left.\mathbf{R}^{\text {cardinality }\left(S_{\alpha}\right)}, \quad \alpha=1, . ., K\right)$ :

$$
\left\{\left(x_{i}\right)_{i=1}^{N} \in \mathbf{R}^{N} ; \sum_{\left\{i ; \Psi_{i} \in V_{\alpha}\right\}} x_{i}=0, \alpha=1, . ., K\right\}
$$

whose $M\left(\epsilon_{0}\right)$-translation is tangent to the range of $M$.

Denote by $f_{a}, a=1, \ldots, N$ the components of $D M$ :

$$
\left.\left.D M(\epsilon)\right|_{\epsilon=\epsilon_{0}} \cdot \tilde{\epsilon}=\left(<f_{a}, \tilde{\epsilon}\right\rangle_{L^{2}}\right)_{a=1}^{N}
$$

Due to the finite dimensionality of our setting we just have to show that the range of $\left.D M(\epsilon)\right|_{\epsilon=\epsilon_{0}}$ has a null orthogonal with respect to $(\mathrm{P})$, that is any vector $\mathbf{k}=$ $\left(k_{a}\right)_{a=1}^{N} \in \mathbf{R}^{N}$ such that

$$
\begin{array}{r}
\sum_{\left\{i ; \Psi_{i} \in V_{\alpha}\right\}} k_{i}=0, \quad \alpha=1, . ., K \\
\sum_{i=1}^{N} k_{i} \cdot<f_{i}, \tilde{\epsilon}>_{L^{2}}=0, \quad \forall \tilde{\epsilon} \in L^{2}([0, T])
\end{array}
$$

is necessary the null vector. Equation (5.6) can also be written

$$
\sum_{i=1} k_{i} \cdot f_{i}(s)=0, \forall 0 \leq s \leq T
$$


The system (5.2) can be written in the integral form:

$c(t)=e^{\int_{0}^{t} \bar{A}+\epsilon_{0} \bar{B}} c(0)+\int_{0}^{t} e^{\int_{s}^{t} \bar{A}+\epsilon_{0} \bar{B}}\left(\epsilon(s)-\epsilon_{0}(s)\right) \bar{B} c(s) d s$

which gives [2] the formula of the (Fréchet) derivative $D_{\epsilon} c\left(\epsilon, C_{0}, t\right)$ of $c\left(\epsilon, C_{0}, t\right)$ with respect to $\epsilon$ computed at $\epsilon(t)=\epsilon_{0}(t)$ :

$\left.D_{\epsilon} c\left(\epsilon, C_{0}, t\right)\right|_{\epsilon=0} \cdot \tilde{\epsilon}=\int_{0}^{t} e^{\int_{s}^{t} \bar{A}+\epsilon_{0} \bar{B}} \tilde{\epsilon}(s) \bar{B} e^{\int_{0}^{s} \bar{A}+\epsilon_{0} \bar{B}} c(0) d s$

Then it is easy to see that

$$
\left.D M(\epsilon)\right|_{\epsilon=0} \cdot \tilde{\epsilon}=\left[2 R e<D_{\epsilon} w(T) \cdot \tilde{\epsilon}\left|\tilde{P}_{a}\right| w(T)>\right]_{a=1}^{N}
$$

so we obtain after some manipulations

$$
f_{a}(s)=2 R e<e^{\int_{s}^{T} \bar{A}+\epsilon_{0} \bar{B}} \bar{B} e^{\int_{T}^{s} \bar{A}+\epsilon_{0} \bar{B}} w(T)\left|\tilde{P}_{a}\right| w(T)>
$$

From 5.7 we obtain that

$$
\left.\sum_{a=1}^{N} k_{a} \frac{d^{k}}{d s^{k}} f_{a}(s)\right|_{s=T}=0
$$

To compute the derivatives $\left.\frac{d^{k}}{d s^{k}} f_{a}(s)\right|_{s=T}$ we make use of a variant of the Campbell - Baker - Hausdorff formula:

$$
e^{-Y \tau} Z e^{Y \tau}=Z+\tau[Y, Z]+\frac{\tau^{2}}{2}[Y,[Y, Z]]+\ldots
$$

Define recursively $a d_{Z}^{i} Y=\left[Y, a d_{Z}^{i-1} Y\right]$ and $a d_{Z}^{0} Y=Z$; we obtain after making use of the hypothesis $\epsilon_{0}(T)=0$ :

$$
R e<a d_{\bar{B}}^{q} \bar{A} w(T)\left|\sum_{a=1}^{N} k_{a} \tilde{P}_{a}\right| w(T)>=0, q \geq 0
$$

The matrix of the operator $\tilde{P}_{a}$ in the basis $\tilde{D}$ is simply $\operatorname{diag}\left\{\delta_{i a}\right\}_{i=1}^{N}$ so the matrix of $\sum_{a=1}^{N} k_{a} \tilde{P}_{a}$ with respect to the basis $D$ is $U^{t}\left(\operatorname{diag}\left\{k_{a}\right\}_{a=1}^{N}\right) U$. By straightforward computations one obtains

$$
a d \frac{q}{B} \bar{A}=\left((-i)^{q+1} \omega_{a b}^{q} B_{a b}\right)_{a, b=1}^{N} .
$$

Note also the general property that when $\omega_{a b}(a<b)$ are all different then the only way to have

$$
\sum_{a<b} \omega_{a b}^{q} r_{a b}=0, q=0,1, \ldots
$$

is when $r_{a b}$ are all zero. Denote $\tilde{w}(T)=U w(T)$ (the coefficients of $\sum_{i} w_{i}(T) \Psi_{i}$ in the base $\left.\tilde{D}\right)$. Using the ingredients above one proves that

$$
\text { If } B_{k l} \neq 0: \sum_{i, j=1}^{N}\left(k_{i}-k_{j}\right) \tilde{w}_{i}(T) \overline{\tilde{w}_{j}(T)} U_{i k} U_{j l}=0
$$

All that remains to be done is to show that the only way to have $(5.5,5.15)$ is when $\mathbf{k}=0$. Note that because any connected component $G_{\alpha}$ has at least cardinality $\left(V_{\alpha}\right)-1$ edges, in $(5.5,5.15)$ there are at least $N$ relations so this is in fact a linear system to solve. We will suppose in all that follows that $G$ has only one connected component; the general case can be reduced to this one due to the commutation relations $\left[\tilde{P}_{a}, P_{b}\right]=0, a=1, \ldots, N, b=1, \ldots, K$.

The remaining of the proof being rather technical so we will only sketch it. Denote $v=U^{t}\left(\operatorname{diag}\left\{k_{j}\right\}_{j=1}^{N}\right) U w(T)$; then equation 5.15 can be written $w_{k}(T) \overline{v_{l}}=\overline{w_{l}(T)} v_{k}$ or, since $w_{i}(T) \neq 0, i=1, \ldots, N$ :

$$
\text { If } B_{k l} \neq 0 \text { then: } \frac{v_{k}}{w_{k}(T)}=\overline{\left(\frac{v_{l}}{w_{l}(T)}\right)}
$$

By the connectivity of $G$ one obtains that there exists $\gamma$ such that for each $i=1, \ldots, N v_{i}=\gamma w_{i}(T)$ or $v_{i}=$ $\bar{\gamma} w_{i}(T)$. If $\gamma$ is real one can infer $\mathbf{k}=0$ by the definition of $v$. If $\gamma$ is not real then divide indexes in two sets $V_{1}$ and $V_{2}$ such that for $i \in V_{1}: v_{i}=\gamma w_{i}(T)$ and for $j \in V_{2}$ : $v_{j}=\bar{\gamma} w_{j}(T)$. One can obtain then a formula for $k_{i}$ :

$k_{i}=\frac{\left(U^{t} v\right)_{i}}{\tilde{w}_{i}(T)}=\frac{\gamma \sum_{j \in V_{1}} U_{i m} w_{m}(T)+\bar{\gamma} \sum_{j \in V_{1}} U_{i m} w_{m}(T)}{\tilde{w}_{i}(T)}$.

By this formula, for $k_{i}$ to be real equation 5.1 from the hypothesis $\mathbb{H C}$ has to be true; if this is not the case then $\gamma$ is real and thus $\mathbf{k}=0$. On the other side if the second assumption of $\mathbb{H C}$ is true then it is easy to prove $k_{a}$ is a constant that does not depend of $a, a=1, \ldots, N$ so by 5.5 we obtain again $\mathbf{k}=0$.

A straightforward application of the theorem above is the following (Thm. 2 from [15]):

Corollary 5.1 (Local exact controllability for populations) Let $d_{0}$ be the population distribution associated to the initial state $C_{0}: d_{0}=\left(\left|c_{0 i}\right|\right)_{i=1, \ldots, N}$. Suppose $d_{0 i} \neq 0, i=1, \ldots, N$ and that the hypothesis $\mathbb{H} \mathbb{A}$ is verified. Then there exists an open neighborhood $D$ of $d_{0}$ on the surface of $\mathbf{R}^{N}$ given by the necessary conditions (4.4) endowed with the canonical topology such that one can exactly reach any population distribution $d$ in $D$ from $C_{0}$. 
Proof: Apply the theorem 5.1 for arbitrary final time $T$ and null electric field $\epsilon(t) \equiv 0$. Since the free evolution of the system preserves the populations of the eigenstates, we obtain for $\tilde{P}_{i}=P_{\Psi_{i}}$ that $\delta_{T}=d_{T}=$ $\left(\left|c_{0 i}\right|\right)_{i=1}^{N}$ so the only hypothesis left to verify is $\mathbb{H C}$. This also is trivial since in this case $U=I$ and for $j$ such that (for instance) $j \in V_{a}^{1}$ the first part of $\mathbb{H} \mathbb{C}$ can be written $\left|<\Psi_{T}, \Psi_{j}>\right|=0$, impossible since $\left|<\Psi_{T}, \Psi_{j}>\right|=\left|c_{0 j}\right| \neq 0$.

Let us also mention for the sake of completeness the global exact controllability result that can be proved [15] using on the one hand the Corollary 5.1 and on the other hand approximate global controllability results (Thm. 3 [15]).

Theorem 5.2 (Global exact controllability) Let $d_{0}$ be the population distribution associated to the initial state $C_{0}: d_{0}=\left(\left|c_{0 i}\right|\right)_{i=1, \ldots, N}$. Under the hypothesis $\mathbb{H} \mathbb{A}$ any population distribution $d=\left(d_{i}\right)_{i=1}^{N}$ such that $d_{i} \neq$ $0, i=1, \ldots, N$ which verifies the necessary conditions (4.4) can be exactly reached from $C_{0}$.

\section{Conclusions}

Controllability of the bilinear quantum systems has been studied in the infinite and finite dimensional settings. The classical control concepts seem to be not very well adapted to the the infinite dimensional case and a negative result has been given as illustration. For the finite dimensional case, positive results have been obtained for exact local controllability of sets of projection-type observables and global controllability has been proven for the particular case when the observables are the populations of eigenstates. Easy to check and intuitively simple to understand necessary and sufficient conditions have been obtained to characterize the attainable set.

Acknowledgements. It is a pleasure to acknowledge helpful talks that we had on this topic with Prof. Yvon Maday (ASCI Laboratory).

\section{References}

[1] A. Assion et al. "Control of Chemical Reactions by Feedback-Optimized Phase-Shaping Femtosecond Laser Pulses" Science vol. 282 (1998) pp. 919-922

[2] J.M.Ball, J.E.Madersen and M.Slemrod, "Controllability for distributed bilinear systems", SIAM J.Control and Optimization, vol 20, No.4, 1982, pp.575597

[3] C. Le Bris, "Control theory applied to Quantum Chemistry: Some tracks", ESAIM : Proceedings, vol. 8, 2000, pp 77-94.
[4] P.Brumer and M.Shapiro, Acc.Chem Res. Vol. 22, p.407, 1989

[5] A.G. Butkovskiy, Yu.I.Samoilenko, "Control of quantum-mechanical processes and systems", Kluwer, 1990

[6] Reinhard Diestel "Graph Theory", 2nd ed. Springer-Verlag, New York, Graduate Texts in Mathematics, Vol. 173, Feb. 2000

[7] C.M. Dion et al., Chem. Phys.Lett 302(1999) 215223

[8] C.M. Dion, A.Keller, O.Atabek \& A.D. Bandrauk, Phys. Rew. A 59(2) 1999, p.1382

[9] Kime K., Appl. Math. Lett. 6 (3) (1993) 11-15.

[10] G.M.Huang, T.J.Tarn \& J.W.Clark, "On the controllability of quantum-mechanical systems", J.Math.Phys 24(1983), p.2608

[11] A.P.Pierce, M.A. Dahleh and H.Rabitz, Phys Rev.A 37, 4950 (1988)

[12] V. Ramakrishna \& al. "Controllability of molecular systems" , Phys. Rew. A, Vol 51, No.2, 1995 pp. 960-966

[13] S.Shi, A.Woody, and H.Rabitz, J.Chem Phys. 88(1988), p.6870

[14] D.J.Tannor and S.A.Rice, J.Chem Phys 83(1985), p. 5013

[15] G. Turinici, "On the controllability of bilinear quantum systems" in M.Defranceschi, C.LeBris (Eds.), Lect. Notes in Chemistry, Springer, (to appear).

[16] Herschel Rabitz, Regina de Vivie-Riedle, Marcus Motzkus, and Karl Kompa "Whither the Future of Controlling Quantum Phenomena?" Science 2000 May 5; 288: 824-828.

[17] W.S.Warren, H.Rabitz and M.Dahleh, "Coherent control of quantum dynamics", Science 259 (1993)

[18] Depending on the problem, one may choose to go beyond this first-order, bilinear term when describing the interaction between the laser and the system $[7,8]$.

[19] For two operators/matrices $T_{1}$ and $T_{2}$ we define $\left[T_{1}, T_{2}\right]=-T_{1} T_{2}+T_{2} T_{1}$.

[20] We refer to [10] for a different view on this issue. Let us point out however that their analysis is done on piecewise constant functions which may not always carry physical meaning for the problem considered; in particular one may prove controllability in this class and notice (by the theorem we present here) that this controllability requires infinite $L^{2}$ norm and therefore infinite laser energy.

[21] This space is given by our model and the functions $\Psi_{i}(x)$ are usually the first eigenfunctions of $H_{0}$ constructed by a prior computation or by a modeling based on observations. 